\title{
ASYMPTOTIC NON-LINEAR NORMAL MODES FOR LARGE-AMPLITUDE VIBRATIONS OF CONTINUOUS STRUCTURES
}

\author{
C. TOUZÉ ${ }^{a, *}$, O. THOMAS ${ }^{a, b}$, A. HUBERDEAU $^{a}$ \\ ${ }^{a}$ ENSTA - UME \\ Chemin de la Hunière, \\ 91761 Palaiseau Cedex, France. \\ ${ }^{b}$ CNAM \\ Structural Mechanics and coupled systems Lab. \\ 2 rue Conté \\ 75003 Paris, France. \\ * Corresponding author: Fax: +33169319997 \\ e-mail adress: touze@ensta.fr
}

Abbreviated title: non-linear normal modes

Total number of pages: 38

Number of figures: 11

Number of tables: 3 


\begin{abstract}
Non-linear normal modes (NNMs) are used in order to derive accurate reduced-order models for large amplitude vibrations of structural systems displaying geometrical nonlinearities. This is achieved through real normal form theory, recovering the definition of a NNM as an invariant manifold in phase space, and allowing definition of new coordinates non-linearly related to the initial, modal ones. Two examples are studied: a linear beam resting on a non-linear elastic foundation, and a non-linear clamped-clamped beam. Throughout these examples, the main features of the NNM formulation will be illustrated: prediction of the correct trend of non-linearity for the amplitude-frequency relationship, as well as amplitude-dependent mode shapes. Comparisons betwen different models -using linear and non-linear modes, different number of degrees of freedom, increasing accuracy in the asymptotic developments- are also provided, in order to quantify the gain in using NNMs instead of linear modes.
\end{abstract}

Keywords: Non-linear normal modes, normal form, geometrical non-linearities. 


\section{INTRODUCTION}

Large amplitude vibrations of continuous structures are described by non-linear Partial Differential Equations (PDEs), which can display a number of different dynamical behaviour [1]. Analysis of such non-linear vibrations is generally performed by substituting a reduced-order model of the lowest possible dimension in place of the original infinitedimensional problem. The usual tools available to perform these model reductions are generally inherited from linear concepts. Galerkin projection onto the linear normal modes (LNMs) is basically the most employed one. A great amount of work deals with accomodations of the basic idea, which consists in finding the best suited ansatz functions so as to separate space and time variables, as well as to limit the number of degrees of freedom (dof) of the system. Among these ones, proper orthogonal decomposition (POD) is an efficient tool, especially when the LNMs of the system are unknown [2-6]. For linear systems, proper orthogonal modes converge to the LNMs as the amount of available data increases $[3,5,7,8]$. The idea is, in essence, linear, and for non-linear systems, POD can be seen as the best linear fit to NNM curved motions in phase space $[3,5]$.

Non-linear normal modes (NNMs), defined as invariant manifold in phase space [9, 10], are aimed at extending the usual linear decomposition to non-linear regimes, hence avoiding the usual pitfalls related to a too severe Galerkin truncation, using for instance a single LNM [11-14]. Due to the loss of invariance of linear eigenspaces in the non-linear range, using a single LNM for non-linear motions omit providing amplitude-dependent mode shapes, and may predict an incorrect trend of non-linearity for the amplitude-frequency response $[13,15]$. This kind of problem has been encountered in circular cylindrical shell vibrations for a long time (see for example [16] and references therein), in non-linear vibrations of buckled beams [17], as well as in suspended cables [18]. 
Several different methods have been proposed during the last decade in order to compute the NNMs (see for example $[9,10,19-24,37]$ and references therein), most of which are based on an asymptotic approach to the non-linear mode. In this paper, real normal form theory is used in order to provide an asymptotic non-linear change of co-ordinates, allowing definition of normal co-ordinates, linked to the representation of dynamics in curved invariant subspaces $[15,24]$. With this formulation, it bears resemblance with the usual decoupling of current use at linear order by diagonalization.

This article is focused on the validation of reduced-order models using NNMs, for application to large amplitude vibrations of continuous structures. Two examples are studied; for each of them, comparisons between different models, using an increasing number of LNMs versus a single NNM, or higher-order single NNM, are compared. It is emphasized that NNM formulation must be used because it overcomes the errors associated to too severe truncations using a single LNM, and for the same complexity at hand (a single oscillator is simulated). Moreover, significative computation time savings are obtained, since a single NNM can replace numerous LNMs. However, it is pointed out that under its asymptotic formulation, NNMs does not seem to provide a reliable and powerful alternative to crude numerical computations using numerous dof, mainly because of the deterioration of the results in strongly non-linear regimes.

\section{THEORETICAL FRAMEWORK}

In this section, the main steps of the computation of asymptotic NNMs through application of real normal form theory are summarized. The interested reader can find the details in $[15,24]$. 
2.1. EQUATIONS OF MOTION

Large-amplitude undamped free vibrations of continuous thin structures subjected to geometrical non-linearities are governed by PDEs of the form:

$$
\ddot{\mathbf{w}}+\mathcal{L}(\mathbf{w}(\mathbf{x}, t))+\mathcal{N}_{2}(\mathbf{w}(\mathbf{x}, t))+\mathcal{N}_{3}(\mathbf{w}(\mathbf{x}, t))=0
$$

with the associated boundary conditions. $\mathbf{w}$ denotes a vector of unknown displacement, $\mathbf{x}$ is the spatial co-ordinates, $t$ represents time, and overdot indicates a derivative with respect to time. $\mathcal{L}$ is a linear spatial operator, which is assumed to be self-adjoint, $\mathcal{N}_{2}$ and $\mathcal{N}_{3}$ are respectively quadratic and cubic non-linear spatial and temporal operators. Only these non-linear terms are kept in usual models such as Von Kármán type equations for beams, arches, plates, shells (see for examples [25]). Throughout the paper, damping will not be considered. The main reason lies in the fact that dynamically important modes are the lightly damped ones, which have very long evolution time constants (see e.g. $[35,36]$ ). Hence the framework considered is for lightly damped structures, fow which the modal damping coefficient $\mu_{p}$ is negligible as compared to the eigenfrequency $\omega_{p}$.

Analysing the linear operator $\mathcal{L}$ with the associated boundary conditions allows one to substitute the problem defined by Eq. (1) for the following:

$$
\forall p=1, \ldots,+\infty: \quad \ddot{X}_{p}+\omega_{p}^{2} X_{p}+\sum_{i=1}^{+\infty} \sum_{j \geq i}^{+\infty} g_{i j}^{p} X_{i} X_{j}+\sum_{i=1}^{+\infty} \sum_{j \geq i}^{+\infty} \sum_{k \geq j}^{+\infty} h_{i j k}^{p} X_{i} X_{j} X_{k}=0
$$

where it has been postulated that:

$$
\mathbf{w}(\mathbf{x}, t)=\sum_{p=1}^{+\infty} X_{p}(t) \Phi_{p}(\mathbf{x}) .
$$

$X_{p}$ is the usual modal co-ordinate, and $\Phi_{p}$ the associated linear mode, whose eigenfrequency is $\omega_{p}$.

Strong reduction of the dimension of problem governed by Eq. (2) is then pursued. For example, it has been usual for a long time to retain only the $p^{\text {th }}$ equation in (2) to study 
non-linear vibrations of the directly excited mode $\Phi_{p}$. Unfortunately, this methodology can produce incorrect results since linear eigenspaces described by modal co-ordinates are not invariant. Thus, residual excitation of non-directly excited modes is present, and neglecting it may lead to erroneous quantitative as well as qualitative results $[11-13,15]$. In other words, linear modal co-ordinates are not the most appropriate ones to describe motions in the non-linear range. Non-linear normal modes, defined as invariant manifold in phase space, are used to remedy this problem.

\subsection{NON-LINEAR CHANGE OF CO-ORDINATES}

Normal form theory allows definition of new co-ordinates that describe invariant manifolds, as well as the non-linear relationship between these new normal co-ordinates and the initial, modal ones. At third order, it reads:

$$
\begin{gathered}
X_{p}=R_{p}+\sum_{i=1}^{N} \sum_{j \geq i}^{N}\left(a_{i j}^{p} R_{i} R_{j}+b_{i j}^{p} S_{i} S_{j}\right) \\
+\sum_{i=1}^{N} \sum_{j \geq i}^{N} \sum_{k \geq j}^{N} r_{i j k}^{p} R_{i} R_{j} R_{k}+\sum_{i=1}^{N} \sum_{j=1}^{N} \sum_{k \geq j}^{N} u_{i j k}^{p} R_{i} S_{j} S_{k}, \\
Y_{p}=S_{p}+\sum_{i=1}^{N} \sum_{j=1}^{N} \gamma_{i j}^{p} R_{i} S_{j}+\sum_{i=1}^{N} \sum_{j \geq i}^{N} \sum_{k \geq j}^{N} \mu_{i j k}^{p} S_{i} S_{j} S_{k}+\sum_{i=1}^{N} \sum_{j=1}^{N} \sum_{k \geq j}^{N} \nu_{i j k}^{p} S_{i} R_{j} R_{k} .
\end{gathered}
$$

$N$ is the number of retained modes in the linear analysis, and is assumed to be as large as wanted. The new normal co-ordinates $\left(R_{p}, S_{p}\right)$ define the $p^{\text {th }}$ NNM $[15,24,26]$. Dynamics in phase space spanned by invariant manifolds is then given, up to third order, by: $\forall p=1, \ldots, N$ :

$\dot{R}_{p}=S_{p}$,

$$
\begin{aligned}
\dot{S}_{p}= & -\omega_{p}^{2} R_{p}-\left(A_{p p p}^{p}+h_{p p p}^{p}\right) R_{p}^{3}-B_{p p p}^{p} R_{p} S_{p}^{2} \\
& -R_{p}\left[\sum_{j>p}^{N}\left[\left(A_{j p j}^{p}+A_{p j j}^{p}+h_{p j j}^{p}\right) R_{j}^{2}+B_{p j j}^{p} S_{j}^{2}\right]+\sum_{i<p}\left[\left(A_{i i p}^{p}+A_{p i i}^{p}+h_{i i p}^{p}\right) R_{i}^{2}+B_{p i i}^{p} S_{i}^{2}\right]\right] \\
& -S_{p}\left[\sum_{j>p}^{N} B_{j p j}^{p} R_{j} S_{j}+\sum_{i<p} B_{i i p}^{p} R_{i} S_{i}\right]
\end{aligned}
$$


Coefficients $\left(A_{i j k}^{p}, B_{i j k}^{p}\right)$ arise from the cancellation of non-resonnant quadratic terms. Eqs. (5) bear now the invariance property, and it is now possible to truncate the system to a low-dimensional subspace without neglecting interactions.

\subsection{SINGLE NON-LINEAR MODE MOTION}

The simplest truncation consists in studying a single mode motion. Restricting the motion on the $p^{\text {th }}$ linear eigenspace leads to a dynamics governed by the following oscillator:

$$
\ddot{X}_{p}+\omega_{p}^{2} X_{p}+g_{p p}^{p} X_{p}^{2}+h_{p p p}^{p} X_{p}^{3}=0
$$

and hence an amplitude-frequency relationship [15]:

$$
\omega_{N L}=\omega_{p}\left(1+\tilde{\Gamma}_{p} a^{2}\right), \quad \text { with } \tilde{\Gamma}_{p}=\frac{1}{8 \omega_{p}^{2}}\left(3 h_{p p p}^{p}-\frac{10 g_{p p}^{p}{ }^{2}}{3 \omega_{p}^{2}}\right),
$$

where $\omega_{N L}$ is the non-linear angular frequency, which depends on the amplitude $a$ considered. If one considers now the motion onto the $p^{t h} \mathrm{NNM}$, the dynamics is now governed by:

$$
\ddot{R}_{p}+\omega_{p}^{2} R_{p}+\left(A_{p p p}^{p}+h_{p p p}^{p}\right) R_{p}^{3}+B_{p p p}^{p} R_{p} S_{p}^{2}=0
$$

and the amplitude-frequency relationship writes:

$$
\omega_{N L}=\omega_{p}\left(1+\Gamma_{p} a^{2}\right), \quad \text { with } \Gamma_{p}=\frac{3\left(A_{p p p}^{p}+h_{p p p}^{p}\right)+\omega_{p}^{2} B_{p p p}^{p}}{8 \omega_{p}^{2}} .
$$

Hence, for different values of the non-linear coefficients $\left(g_{i j}^{p}, h_{i j k}^{p}\right)$, the trend of nonlinearity predicted by Eq. (7) or (9) may be opposite [15].

In the following, this prediction will be confirmed on a continuous system possessing quadratic and cubic non-linearities. Then, comparisons between the number $N$ of LNMs retained (from Eq. 2) and the number $M$ of NNMs retained after truncation in Eq. (5) are performed, in order to test the accuracy obtained versus the computing time saved by simulating a few NNMs. 
FOUNDATION

3. EXAMPLE 1: A LINEAR BEAM ON A NON-LINEAR ELASTIC

3.1. EQUATIONS OF MOTION

A linear Euler-Bernoulli beam, hinged at its two ends, and resting on a non-linear elastic foundation with quadratic and cubic distributed non-linearities, is considered. In nondimensional form, the undamped transverse vibrations are governed by [13]:

$$
\frac{\partial^{2} w}{\partial t^{2}}+\frac{\partial^{4} w}{\partial x^{4}}+\alpha_{2} w^{2}+\alpha_{3} w^{3}=0
$$

$w(x, t)$ is the transverse displacement, $\alpha_{2}$ and $\alpha_{3}$ are two parameters. The considered boundary conditions write:

$$
w(x, t)=0, \quad \frac{\partial^{2} w(x, t)}{\partial x^{2}}=0 \quad \text { for } \quad x=0,1 .
$$

The linear analysis provides the eigenmodes as well as the eigenfrequencies:

$$
\begin{aligned}
& \Phi_{n}(x)=\sqrt{2} \sin (n \pi x), \\
& \omega_{n}=n^{2} \pi^{2} .
\end{aligned}
$$

Denoting $X_{p}$ the modal co-ordinate associated to the $p^{t h}$ linear mode, the projection yields the following problem, equivalent to Eq. (10) for $N \rightarrow+\infty: \forall p=1, \ldots, N$ :

$$
\ddot{X}_{p}+\omega_{p}^{2} X_{p}+\sum_{i, j=1}^{N} \tilde{g}_{i j}^{p} X_{i} X_{j}+\sum_{i, j, k=1}^{N} \tilde{h}_{i j k}^{p} X_{i} X_{j} X_{k}=0
$$

where:

$$
\tilde{g}_{i j}^{p}=\alpha_{2} \int_{0}^{1} \Phi_{i}(x) \Phi_{j}(x) \Phi_{p}(x) \mathrm{d} x, \quad \tilde{h}_{i j k}^{p}=\alpha_{3} \int_{0}^{1} \Phi_{i}(x) \Phi_{j}(x) \Phi_{k}(x) \Phi_{p}(x) \mathrm{d} x .
$$

Eq. (14) is then made similar to Eq. (2) by considering the symetries of the nonlinear quadratic and cubic terms (e.g. for $j>i, g_{i j}^{p}=\tilde{g}_{i j}^{p}+\tilde{g}_{j i}^{p}$ and $g_{j i}^{p}=0$ ).

Due to the particular expression of the eigenfrequencies, numerous internal resonances are present. They are taken into account in the model by keeping in the normal form 
resonant terms that cannot be cancelled through the non-linear change of co-ordinates. Table 1 summarizes the internal resonances for the ten first linear modes, as well as the associated coefficients in the dynamical equations. Some of these coefficients are equal to zero (right column of Table 1), which means that the internal resonance does not exist since the corresponding coupling terms are not present. In particular, second-order internal resonances (linked to the quadratic terms), are not present. The cubic coefficients that have to stay in the normal form are also given, with the usual notation: if $h_{i j k}^{p}$ is not equal to zero, then a term of the form $R_{i} R_{j} R_{k}$ has to be kept in $p^{t h}$ equation of the normal form (Eq. (5)). As already pointed out in [15], these coefficients doesn't involve invariant-breaking terms. Hence single NNM motions can be studied.

\subsection{NUMERICAL SIMULATIONS}

Regions of hardening or softening behaviour in the parameter plane $\left(\alpha_{2}, \alpha_{3}\right)$ are different if one considers a single linear mode or a single non-linear mode [15]. Fig. 1 shows these regions for mode 2 and 3 (the difference between the two regions is negligible for mode 1). For mode 2, a single linear mode truncation predicts that the non-linearity will be of the hardening type for any positive values of $\left(\alpha_{2}, \alpha_{3}\right)$ since $g_{22}^{2}=0$. Considering the second NNM shows that there is a region in the parameter plane for which the behaviour will be of the softening type.

Numerical simulations are conducted in order to verify these statements. In each case, a reference solution is computed by simulating Eq. (14) for a large number $N$ of modes retained. In practice, it has been found that from 6 LNMs, the computed vibration signals are equivalent. To get confidence in the reference solution, $N=10 \mathrm{LNMs}$ are used. A time-integration fourth-order Runge-Kutta scheme is used, with sufficient accuracy in the time step and a total length equal to ten periods. 
For mode 2 and 3, three different solutions are compared: (i) the reference solution, (ii) the NNM solution, and (iii) the single LNM solution. In each case, numerical solutions are computed for increasing values of initial conditions. The frequency is then estimated by measuring the time gap between two zero crossings, mean valued on ten periods. For (ii) and (iii), analytical solutions derived from Eqs (9) and (7) are also shown. Figure 2 summarizes the results with the so-called backbone curves.

For each values of the parameters, the NNM solution predicts the correct trend of nonlinearity, whereas the single linear mode solution can give erroneous qualitative results. This is a confirmation of earlier results obtained for a two d.o.f. system [15]. It clearly shows that single-NNM solution have to be considered if one wants to obtain results with a single oscillator that predicts the correct trend of non-linearity for a continuous structure. These results can be applied for example to circular cylindrical shells vibrations where the correct softening type behaviour is obtained by considering a large number of linear modes $[16,27]$. Finally, the single-NNM solution fails to predict the correct frequency for high vibration amplitudes. This is a typical feature of the present formulation of the NNMs, which relies upon an asymptotic development that diverges from the exact solution upon a certain threshold.

It has been shown that a single NNM is more accurate that a single linear mode truncation. The main reason is that the NNM formulation, based upon invariant manifolds, takes into account the bending of the phase space; and thus the effect of a priori non-retained modes in the truncation is not neglected. The question of how many linear modes are needed to obtain the same accuracy than that obtained with a single NNM is now adressed. Numerous simulations have been conducted for mode 2 and 3. An interesting one is 
shown on Figure 3, with $\alpha_{2}=20$ and $\alpha_{3}=3$. Backbone curves for a single NNM, a single LNM, two LNMs and the reference solution, are represented. For moderately large amplitude, the 2 LNMs simulation is as accurate as the single NNM for the frequency estimation. Single NNM solution becomes incorrect for $a>1.5$, amplitude for which the curvature of the backbone curve changes.

Generally speaking, it has been found that a simulation with 3 LNMs is always as good, or better, as a single NNM, mainly because the approximation is spatially more precise. Figure 4 shows the vibration of the beam at its center, for an inital amplitude of $R_{2}(t=$ $0)=1$. The single linear mode solution is not represented because it doesn't predict the existence of a drift in the solution, and thus the displacement at this point is zero [15]. The NNM solution is more precise in amplitude than the two LNMs simulation, hence resulting in a smaller global error for the beam displacement.

\section{EXAMPLE 2: A NON-LINEAR BEAM}

\subsection{GOVERNING EQUATIONS}

A clamped clamped beam vibrating with moderately large amplitude is considered. In non-dimensional form, the transverse displacement $w$, which has been choosen such that $w=1$ in the model means a displacement equal to the thickness $h$, is solution of:

$$
\frac{\partial^{2} w}{\partial t^{2}}+\frac{\partial^{4} w}{\partial x^{4}}-\frac{\varepsilon}{2}\left[\int_{0}^{1}\left(\frac{\partial w}{\partial x}\right)^{2} d x\right] \frac{\partial^{2} w}{\partial x^{2}}=0
$$

with boundary conditions:

$$
w(0, t)=w(1, t)=w_{, x}(0, t)=w_{, x}(1, t)=0 .
$$

The parameter $\varepsilon$ is equal to: $\varepsilon=\frac{A h^{2}}{I}$, where $A$ and $I$ are the cross-section area and moment of inertia. Linear normal modes write:

$$
\Phi_{k}(x)=K\left[\cos \beta_{k} x-\cosh \beta_{k} x+\frac{\sin \beta_{k}+\sinh \beta_{k}}{\cos \beta_{k}-\cosh \beta_{k}}\left(\sin \beta_{k} x-\sinh \beta_{k} x\right)\right],
$$


where $K$ is such that $\int_{0}^{1} \Phi_{k}(x)^{2} d x=1$. Natural angular frequencies are solutions of $\cos \beta_{k}=\frac{1}{\cosh \beta_{k}}$, where $\omega_{k}=\beta_{k}^{2}$. The PDE (16) is then projected onto the natural modes basis, and symmetric non-linear cubic terms are gathered. The resulting temporal problem to solve reads: $\forall p=1, \ldots, N$ :

$$
\ddot{X}_{p}+\omega_{p}^{2} X_{p}+\varepsilon \sum_{i=1}^{N} \sum_{j \geq i}^{N} \sum_{k \geq j}^{N} \breve{h}_{i j k}^{p} X_{i} X_{j} X_{k}=0 .
$$

In the following, a squared cross-section is assumed, so that $\varepsilon=12$. The number $N$ of LNMs retained for the reference solution is equal to 20. A few values of the coefficients $\breve{h}_{i j k}^{p}$ are given in Table 2, showing that the non-linear terms have very large magnitudes in the following simulations.

\subsection{MODE SHAPES}

An interesting feature of the NNM formulation is its ability to predict amplitude-dependent mode shapes. These are shown on Figure 5 for the first three modes and for different amplitudes. The corresponding linear mode shape is also shown for comparison. These figures have been computed for a zero velocity $\left(S_{p}=0\right)$, and a maximum of the corresponding normal co-ordinate $R_{p}$, for $p=1,2,3$. The same kind of dependence on amplitude has been found by applying a completely different method, the hierarchical finite element method, to the same problem [28].

When oscillating along a single NNM, the mode shape vary continuously with time. This is illustrated on Figure 6, where the mode shapes for the first two modes are represented at three different instant of time, choosen in the first quarter-period of motion. 


\subsection{HIGHER-ORDER APPROXIMATION OF THE DYNAMICS}

One major drawback of the formulation of the NNMs used in this study is that it relies upon an asymptotic development for computing the geometry of the phase space, as well as the normal dynamics. Their formal expressions, up to order three, are given by Eqs. (5) and (4).

For a problem with quadratic and cubic non-linearity, the order-three normal dynamics allows correction due to the bending of the phase space contained within the quadratic terms. This is responsible for the difference of hardening or softening behaviour detected when considering a single linear mode or a single NNM (see Eqs (6) and (8)).

For a problem with only cubic non-linearity like the non-linear beam, a single LNM and a single NNM up to order three are governed by the same oscillator. Hence the differences between the two truncation will be only visible in the geometry of the manifold, and thus only in the spatial computed response $w(\mathbf{x}, t)$ and not in the approximated frequency. To obtain better accuracy in the computed frequency, a higher-order approximation of the dynamics onto the invariant manifold is seeked.

Deriving the general order-five equations for the non-linear change of co-ordinates as well as the normal dynamics has not been realized because of the complexity involved in the calculations. However, considering a motion involving only the $p^{\text {th }}$ NNM allows one to compute easily the dynamics up to order five, for an initial problem with only cubic non-linearities. Assume that the $p^{t h}$ NNM only is involved in the vibration. Thus $\forall k \neq$ $p, R_{k}=0$. We can then substitute for:

$$
\begin{gathered}
\forall k \neq p: \quad X_{k}=r_{p p p}^{k} R_{p}^{3}+u_{p p p}^{k} R_{p} S_{p}^{2}, \\
X_{p}=R_{p},
\end{gathered}
$$

in the original equations: Eq. (2) without quadratic terms. As we are interested only in 
the dynamics up to order five, the third-order development (Eq. (20)) is sufficient. Indeed, one has just to retain the quintic resonant terms introduced by the cubic non-linear change of coordinates to obtain the dynamics onto the manifold. it is a general feature of these developments that the level of accuracy obtained for the dynamics is one order higher than that of the change of coordinates. This leads to the following equation for a single NNM dynamics:

$$
\ddot{R}_{p}+\omega_{p}^{2} R_{p}+h_{p p p}^{p} R_{p}^{3}+\Xi_{p} R_{p}^{5}+\Upsilon_{p} R_{p}^{3} S_{p}^{2}=0
$$

where:

$$
\begin{aligned}
& \Xi_{p}=\sum_{k>p}^{N} r_{p p p}^{k} h_{p p k}^{p}+\sum_{k<p} r_{p p p}^{k} h_{k p p}^{p}, \\
& \Upsilon_{p}=\sum_{k>p}^{N} u_{p p p}^{k} h_{p p k}^{p}+\sum_{k<p} u_{p p p}^{k} h_{k p p}^{p} .
\end{aligned}
$$

As the two supplementary quintic terms in Eq. (21) are resonant, they can't be eliminated through a non-linear change of co-ordinate. Thus Eq. (21) is the accurate dynamics, up to order five, for a single NNM motion.

It is also possible to derive an approximation of the dynamics onto the manifold up to order seven, by retaining the order seven terms in the abovepresented calculation. But only an approximation will be obtained. For deriving the right equation governing the dynamics for a single NNM up to order seven, one has to compute the complete calculation up to order five, because the elimination of non-resonant quintic terms through a fifth order change of co-ordinates will reintroduce other seventh-order terms. However, this seventhorder approximation has been computed and tested in the simulations.

Figure 7 shows a simulation realized for a motion initiated along the first NNM with initial conditions $R_{1}=0.8, S_{1}=0$. The vibration at the quarter of the beam is shown, and for different order of simulation. The reference solution is obtained by simulating the complete system with $N=20$ linear modes and a numerical Runge-Kutta time integration 
scheme. One can see that the third order NNM predicts a higher frequency than the reference solution. The seventh-order approximation is the most accurate, and is used in the subsequent simulations.

Numerous simulations have then been conducted for testing the accuracy of a single NNM simulation versus an increasing number of linear modes retained in the original dynamics. Figure 8 shows the results of a simulation conducted with initial condition $R_{1}=$ $0.8, S_{1}=0$, and for two positions on the beam : vibration at center (first row) and at one tenth of the beam (second row). An estimation of the error committed has been computed with the following criterion:

$$
e=\frac{\left\|w^{r e f}(\mathbf{x}, t)-w^{m o d}(\mathbf{x}, t)\right\|_{2}}{\left\|w^{r e f}(\mathbf{x}, t)\right\|_{2}}
$$

where $w^{r e f}(\mathbf{x}, t)$ is the reference solution and $w^{\text {mod }}(\mathbf{x}, t)$ refers to the solution obtained with one reduced-order model : single NNM, single LNM or few number of LNMs. The $\|\cdot\|_{2}$ is the euclidean norm, computed for a given duration of 1 of adimensional time, and for different position $\mathbf{x}$ on the beam. The results are given in Table 3. The NNM solution is very accurate until $R_{1}=1$ is reached, and then becomes worst than the 3 LNMs solution. A simulation with $R_{1}=1.4$ is shown on Figure 9, observed at $\mathbf{x}=0.05$, showing that for too large amplitudes, the seventh-order NNM fails to predict the correct frequency. Generally speaking, and for $R_{1} \leq 1$, it has been found that the 4 LNMs solution is as accurate as the NNM simulation, and the 6 LNMs solution is undistinguishable from the reference solution.

\subsection{MULTI-MODE SOLUTIONS}

Simulations with initial conditions involving two nonlinear modes have also been conducted. As the complete change of co-ordinates allowing to pass from the modal coordinates to the normal ones is provided, these kind of simulations are directly available, 
which constitutes one of the main advantage of using Normal Form theory. This is contrary to the methods based on center manifold reduction, where the reduction to a fourdimensional invariant manifold has first to be computed $[29,30]$. Due to the large number of terms that have to be retained, the fifth-order normal dynamics for two NNMs have not been calculated. Hence the simulations presented are up to cubic order.

Figure 10 shows the result obtained for an initial condition $R_{1}=0.5, R_{2}=0.5$, and no initial velocities. As the precision is at third order, the NNM simulation doesn't predict very well the frequency. And the four LNMs simulations give much more accurate results. Numerous simulations have been performed for different inital values and the gain in using NNMs instead of linear modes has never been found to be very important.

\section{DISCUSSION}

The aim of the present study was to run several numerical simulations in order to quantify the gain of using NNMs as reduced-order models of large-scale structural systems. The long-term goal of this research is to define efficient and accurate methods that improve significantly finite element codes for non-linear vibrations, through definition of non-linear modal analysis/synthesis.

In this paper, asymptotic non-linear normal modes, defined as invariant manifold in phase space, and computed with normal form theory, are used. This formulation provides a theoretical framework which allows thinking the NNMs as a natural extension of the traditional linear decoupling through diagonalization, mainly because a global non-linear change of co-ordinates is defined. As shown on the diagram represented on figure (11), the reduction of the phase space dimension through distinction between master and slave co-ordinates allows definition of a clean framework for non-linear modal analysis/synthesis.

Simulations have shown that NNM formulation must be used for performing severe trun- 
cation in the PDEs. In any case, using a single NNM is much better than using a single LNM. It allows prediction of the correct trend of non-linearity for a problem with quadratic and cubic coefficients. For the non-linear beam problem, higher-order asymptotic developments enables to recover a good estimation of the frequency. Acquiring precision in the change of co-ordinates has to be related with the accurate location of the invariant manifolds, and thus with slight spatial differences in the computed deflections. Precision in the normal dynamics (Eq. (5)) is related to dynamical information, and, at first glance, to the estimation of the non-linear oscillation frequency.

Unfortunately, the cubic order asymptotic development is not enough accurate and the results deteriorates at higher vibration amplitudes. Slight improvements are possible for a better approximation of the normal dynamics, as it has been shown on the non-linear beam. But deriving general higher-order expressions is messy and algebraically quite intractable.

The question of how many linear modes are needed in order to obtain the same level of accuracy than that obtained with a single NNM has also been adressed. The two examples studied showed that $3 \mathrm{LNMs}$ is better for example 1 , and 4 for example 2 . The same number of modes seems necessary in the case of suspended cables [18]. This number should be bigger for two-dimensional structures, as it can be inferred from recent studies with large number of dofs on circular shell vibrations (where seven modes were kept to obtain accurate results) [27], or circular cylindrical panels (11 LNMs were necessary) [31]. But once again, at very high amplitudes, the results deteriorates and using a single asymptotic third order NNM is meaningless. These results corroborates earlier results obtained by Boivin, Pesheck et al. [32, 29, 30].

The computational time saved by using these reduced-order models is nonetheless significant. But at this point, it does not seem to be enough time savings for large-scale 
crude numerical simulations. Hence the method has to be enhanced to become a powerful tool in non-linear structural dynamics. A solution would be to break away from asymptotic developments. Some significative improvements have been realized in this direction with a formulation based on center manifold theorem [22]. A precize non-linear change of co-ordinate should thus be seeked, but it does not seem straightforward since Poincaré's theorem, which is the cornerstone of normal form theory, is proven with an asymptotic development (see e.g. $[33,34]$ ). As a conclusion, the asymptotic formulation shows that NNMs must be used, but also that significative improvements are needed in order to deliver its complete potential, which is here just lightly touched. 


\section{REFERENCES}

1. A. H. Nayfeh. Nonlinear interactions: analytical, computational and experimental methods. Wiley series in nonlinear science, New-York, 2000.

2. P. Holmes, J. L. Lumley and G. Berkooz. Turbulence, Coherent structures, Dynamical systems and Symmetry. Cambridge University Press, 1996.

3. B. F. Feeny and R. Kappagantu. On the physical interpretation of proper orthogonal modes in vibrations. J Sound Vib 1998; 211(4):607-616.

4. B. F. Feeny. On proper orthogonal co-ordinates as indicators of modal activity. J Sound Vib 2002; 255(5):805-817.

5. G. Kerschen and J. C. Golinval. Physical interpretation of the proper orthogonal modes using the singular value decomposition. J Sound Vib 2002; 249(5):849-865.

6. M. Amabili, A. Sarkar and M. P. Païdoussis. Reduced-order models for non-linear vibrations of cylindrical shells via the proper orthogonal decomposition method. J Fluids Struct 2003; 18:227-250.

7. W. Z. Lin, K. H. Lee, P. Lu, S. P. Lim and Y. C. Liang. The relationship between eigenfunctions of Karhunen-Loève decomposition and the modes of distributed parameter vibration system. J Sound Vib 2002; 256(4):791-799.

8. Y. C. Liang, H. P. Lee, S. P. Lim, W. Z. Lin, K. H. Lee and C. G. Wu. Proper orthogonal decomposition and its applications-Part I: theory. J Sound Vib 2002; 252(3):527-544.

9. S. W. Shaw and C. Pierre. Normal Modes for non-linear vibratory systems. J Sound Vib 1993; 164(1):85-124.

10. S. W. Shaw and C. Pierre. Normal Modes of vibration for non-linear continuous systems. J Sound Vib 1994; 169(3): 319-347.

11. A. H. Nayfeh, J. F. Nayfeh and D. T. Mook. On methods for continuous systems with quadratic and cubic nonlinearities. Nonlinear Dynamics 1992; 3:145-162.

12. M. Pakdemirli, S. A. Nayfeh and A. H. Nayfeh. Analysis of one-to-one autoparametric resonances in cables. Discretization vs. direct treatment. Nonlinear Dynamics 1995; 8:65-83.

13. A. H. Nayfeh and W. Lacarbonara. On the discretization of distributed-parameter systems with quadratic and cubic nonlinearities. Nonlinear Dynamics 1997; 13:203-220.

14. A. H. Nayfeh. Reduced-order models of weakly nonlinear spatially continuous systems. Nonlinear Dynamics 1998; 16:105-125.

15. C. Touzé, O. Thomas and A. Chaigne. Hardening/softening behaviour in non-linear oscillations of structural systems using non-linear normal modes. to appear in J Sound Vib. 
16. M. Amabili, F. Pellicano and M. P. Païdoussis. Nonlinear vibrations of simply supported, circular cylindrical shells, coupled to quiescent fluid. J Fluids Struct 1998; 12:883-918.

17. W. Lacarbonara. A theoretical and experimental investigation of nonlinear vibrations of buckled beams. PhD thesis, Virginia polytechnic institute and state university, 1997.

18. H. N. Arafat and A. H. Nayfeh. Non-linear responses of suspended cables to primary resonance excitation. J Sound Vib 2003; 266(2):325-354.

19. A. F. Vakakis, L. I. Mikhlin, Y. V. Philipchuck and A. A. Zevin. Normal modes and localization in nonlinear systems. New-York: John Wiley and Sons, 1996.

20. L. Jézéquel and C. H. Lamarque. Analysis of non-linear dynamical systems by the normal form theory. J Sound Vib 1991; 149(3):429-459.

21. A. H. Nayfeh and S. A. Nayfeh. On nonlinear modes of continuous systems. Trans. ASME, J of Vib Acoust 1994; 116:129-136.

22. E. Pesheck, C. Pierre and S. W. Shaw. A new Galerkin-based approach for accurate non-linear normal modes through invariant manifolds. J Sound Vib 2002; 249(5):971-993.

23. M. E. King and A. F. Vakakis. An energy-based formulation for computing nonlinear normal modes in undamped continuous systems. J Vib Acoust 1994; 116:332-340.

24. C. Touzé. A normal form approach for non-linear normal modes. Internal publication of the LMA 2003; vol. 156:15-38 (ISSN: 1159-0947, ISBN:2-909669-20-3), Marseille, France.

25. A. H. Nayfeh and D. T. Mook. Nonlinear oscillations. John Wiley and Sons, 1979.

26. C. Touzé and O. Thomas. Determination of non-linear normal modes for conservative systems. Proc. of the Ninth ICSV, Orlando, paper No. 70, 2002.

27. M. Amabili, F. Pellicano and M. P. Païdoussis. Non-linear dynamics and stability of circular cylindrical shells containing flowing fluid, part II: large-amplitude vibrations without flow. J Sound Vib 1999; 228(5):1103-1124.

28. P. Ribeiro and M. Petyt. Non-linear vibration of beams with internal resonance by the hierarchical finite-element method. J Sound Vib 1999; 224(4):591-624.

29. E. Pesheck. Reduced-order modeling of nonlinear structural systems using nonlinear normal modes and invariant manifolds. PhD thesis, University of Michigan, 2000.

30. E. Pesheck and C. Pierre. A global methodology for the modal reduction of large nonlinear systems containing quadratic and cubic nonlinearities. Proc. of DETC'97, Sacramento, 1997.

31. M. Amabili and M. Pellegrini. Nonlinear vibrations of circular cylindrical panels. Proc. of the Second MIT Conference, K.J. Bathe Editor, pp. 50-53, June 2003.

32. N. Boivin, C. Pierre and S. Shaw. Non-linear normal modes, invariance, and modal dynamics approximations of non-linear systems. Nonlinear Dynamics 1995; 8:315-346. 
33. V. I. Arnold. Chapitres supplémentaires de la théorie des équations différentielles ordinaires. Mir-Librairie du Globe, 1980.

34. A. D. Brjuno. Analytical form of differential equations. Trans. Moscow Math. Soc. 1971; 25:131-288.

35. J. Guckenheimer, P. J. Holmes. Non-linear oscillations, dynamical systems and bifurcation of vector fields. Spreinger, new-York, 1986.

36. P. Manneville. Dissipative structures and weak turbulence. Academic Press, 1990.

37. R. Bouc, S. Bellizzi. Une nouvelle approche pour l'existence et le calcul des modes nonlinéaires. Internal publication of the LMA 2003; vol. 156:38-58 (ISSN: 1159-0947, ISBN:2909669-20-3), Marseille, France. 
Caption for tables

Table 1. Internal resonance relations between the ten first eigenfrequencies, and associated non-linear coefficients; for the linear beam resting on a non-linear foundation.

Table 2. Values of the coefficients of the non-linear beam problem for modes 1, 2 and 3. To be equal to the $h_{i j k}^{p}$ defined in Eq. (2), these values have to be multiplied by $\varepsilon$.

Table 3. Errors committed with several different simulations, for increasing values of the non-linearity. 
Caption for figures

Figure 1. Hardening/softening regions in the parameter plane $\left(\alpha_{2}, \alpha_{3}\right)$ for modes 2 and 3. $H / S$ refers to hardening/softening regions for a single NNM, $h / s$ for a single LNM. $\diamond$ : values of the parameters retained for Figure 2.

Figure 2. Backbones curves for different values of the parameters $\left(\alpha_{2}, \alpha_{3}\right) . \diamond$ : reference solution, $\triangle$ : single NNM simulation, $\circ$ : single LNM simulation. Analytical first-order solutions are also represented, plain line for the NNM (Eq. (9)), dash-dotted for single LNM (Eq. (7)). (a): $\alpha_{2}=12, \quad \alpha_{3}=.5$; (b): $\alpha_{2}=14, \quad \alpha_{3}=1.8$; (c): $\alpha_{2}=14, \quad \alpha_{3}=0.001$; (d): $\alpha_{2}=5, \alpha_{3}=0.03$.

Figure 3. Backbone curves for $\alpha_{2}=20$ and $\alpha_{3}=3$, and for different truncations.

Figure 4. Displacement of the beam at its center. $\alpha_{2}=20$ and $\alpha_{3}=3$. Initial condition : $R_{2}=1, S_{2}=0 . w=1$ in the model means a real displacement equal to the thickness $h$ of the beam.

Figure 5. Non-linear Mode shapes at different vibration amplitudes, for mode 1, 2 and 3 (plain line). Dash-dotted lines represent the corresponding linear mode.

Figure 6. Evolution of the mode shape during a quarter-period of motion. Initial condition for mode $1: R_{1}=1.5$, for mode $2: R_{2}=1$.

Figure 7. Displacement of the beam at $x=0.25$ and for an initial condition taken along the 
first NNM : $R_{1}=0.8, S_{1}=0$. Different order of accuracy in the asymptotic development for the dynamics are shown.

Figure 8. Displacement of the beam at its center (first row) and at $x=0.1$ (second row), for initial condition $R_{1}=0.8, S_{1}=0$. An increasing number of LNM versus a single NNM are represented. The solution with five linear modes is superposed to the reference solution (obtained with $N=20 \mathrm{LNMs}$ ).

Figure 9. Displacement of the beam at $x=0.05$ for an initial condition $R_{1}=1.4$. In this case, the peak-to-peak displacement at center to 4 .

Figure 10. Displacement of the beam at its center (first row) and at $x=0.1$ (second row), for initial condition $R_{1}=0.5, R_{2}=0.5$.

Figure 11. Non-linear modal analysis/synthesis through NNMs and real normal form. 


\begin{tabular}{ll}
\hline order-two internal resonances & associated coefs. \\
\hline$\omega_{5}=\omega_{3}+\omega_{4}$ & $\equiv 0$ \\
$\omega_{10}=\omega_{6}+\omega_{8}$ & $\equiv 0$ \\
\hline order-three internal resonances & associated coefs. \\
\hline$\omega_{1}=\omega_{4}+\omega_{7}-\omega_{8}$ & $\equiv 0$ \\
$\omega_{1}=2 \omega_{5}-\omega_{7}$ & $\equiv 0$ \\
$\omega_{2}=\omega_{6}+\omega_{7}-\omega_{9}$ & $\equiv 0$ \\
$\omega_{3}=\omega_{1}+2 \omega_{2}$ & $h_{122}^{3}, h_{223}^{1}, h_{123}^{2}$ \\
$\omega_{6}=\omega_{2}+2 \omega_{4}$ & $h_{446}^{2}, h_{246}^{4}, h_{244}^{6}$ \\
$\omega_{7}=\omega_{2}+\omega_{3}+\omega_{6}$ & $h_{236}^{7}, h_{367}^{2}, h_{267}^{3}, h_{237}^{6}$ \\
$\omega_{9}=\omega_{1}+\omega_{8}+\omega_{4}$ & $\equiv 0$ \\
$\omega_{9}=\omega_{3}+2 \omega_{6}$ & $h_{669}^{3}, h_{369}^{6}, h_{366}^{9}$ \\
$\omega_{9}=2 \omega_{4}+\omega_{7}$ & $\equiv 0$ \\
\hline
\end{tabular}

Table 1. 


\begin{tabular}{l|lll}
\hline & $\mathbf{1}$ & $\mathbf{2}$ & $\mathbf{3}$ \\
\hline $\mathbf{1}$ & $\breve{h}_{111}^{1}=75.7$ & $\breve{h}_{112}^{1}=1.4 \mathrm{e}-5$ & $\breve{h}_{113}^{1}=-179.6$ \\
& & $\breve{h}_{122}^{1}=283.3$ & $\breve{h}_{133}^{1}=703.1$ \\
& & $\breve{h}_{222}^{1}=1.8 \mathrm{e}-5$ & $\breve{h}_{333}^{1}=-481.2$ \\
$\mathbf{2}$ & $\breve{h}_{111}^{2}=4.8 \mathrm{e}-6$ & $\breve{h}_{222}^{2}=1060.3$ & $\breve{h}_{223}^{2}=1.8 \mathrm{e}-5$ \\
& $\breve{h}_{112}^{2}=283.3$ & & $\breve{h}_{233}^{2}=2277.3$ \\
& $\breve{h}_{122}^{2}=5.4 \mathrm{e}-5$ & & \\
& & & \\
$\mathbf{3}$ & $\breve{h}_{133}^{2}=1.3 \mathrm{e}-5$ \\
& $\breve{h}_{113}^{3}=-59.9$ & $\breve{h}_{222}^{3}=6.1 \mathrm{e}-6$ & $\breve{h}_{333}^{3}=4891.1$ \\
& $\breve{h}_{133}^{3}=-1443.6$ & $\breve{h}_{2233}^{3}=2277.3$ & \\
\hline
\end{tabular}

Table 2. 


\begin{tabular}{l||ll|ll|lll||l}
\hline$R_{1}$ & NNM, & NNM, & 1 & LNM, 1 & LNM, & LNMs, 3 & LNMs, & $\max (\mathbf{w})$ \\
& $\mathrm{x}=.5$ & $\mathrm{x}=.1$ & $\mathrm{x}=.5$ & $\mathrm{x}=.1$ & $\mathrm{x}=.5$ & $\mathrm{x}=.1$ & \\
\hline 0.5 & 0.0033 & 0.0053 & 0.0462 & 0.0662 & 0.0056 & 0.0125 & 0.78 \\
0.7 & 0.0189 & 0.0245 & 0.1409 & 0.1595 & 0.0193 & 0.0281 & 1.10 \\
0.8 & 0.0354 & 0.0420 & 0.2189 & 0.2345 & 0.0351 & 0.0417 & 1.24 \\
1 & 0.1047 & 0.1207 & 0.3983 & 0.3995 & 0.0698 & 0.0803 & 1.54 \\
1.2 & 0.2289 & 0.2629 & 0.5743 & 0.5557 & 0.1291 & 0.1414 & 1.87 \\
\hline
\end{tabular}

Table 3. 

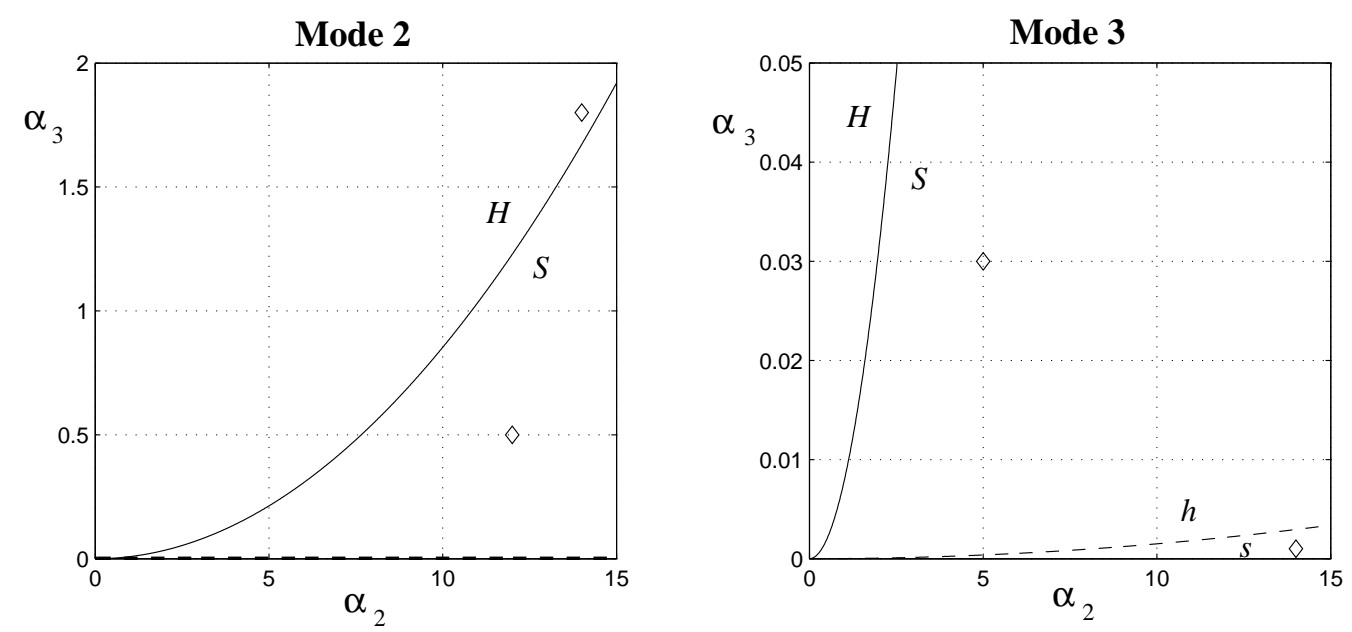

Figure 1. 
Mode 2

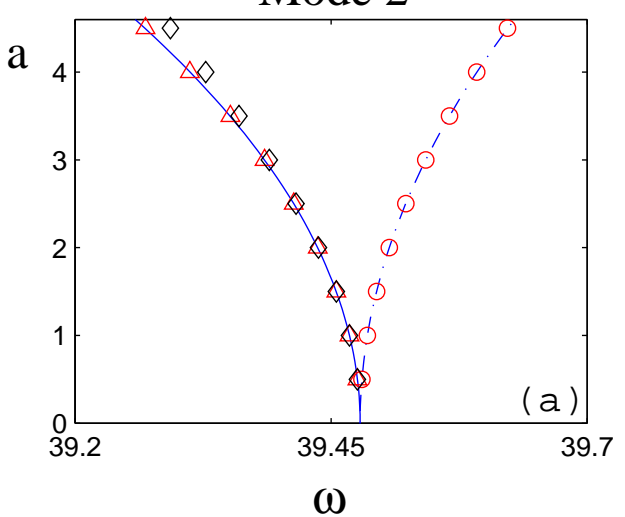

a

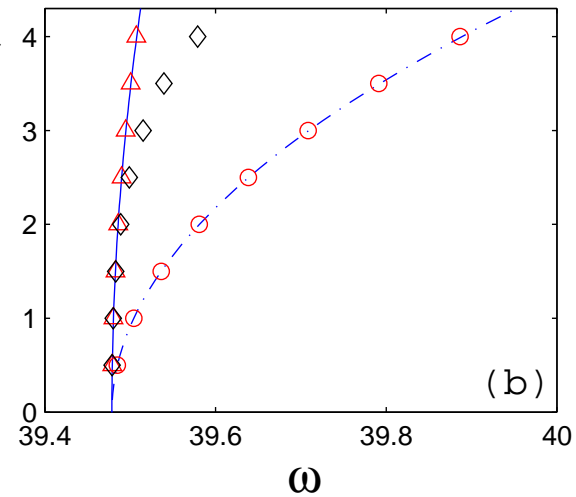

Mode 3

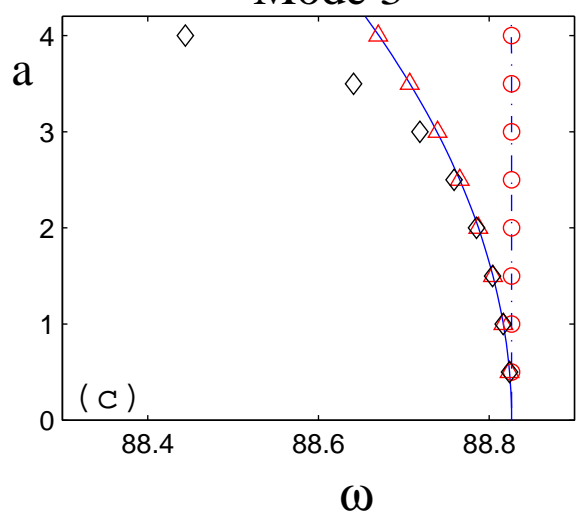

a

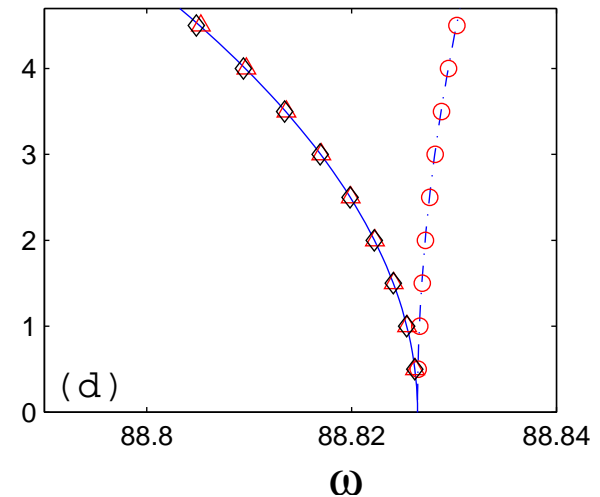

Figure 2. 


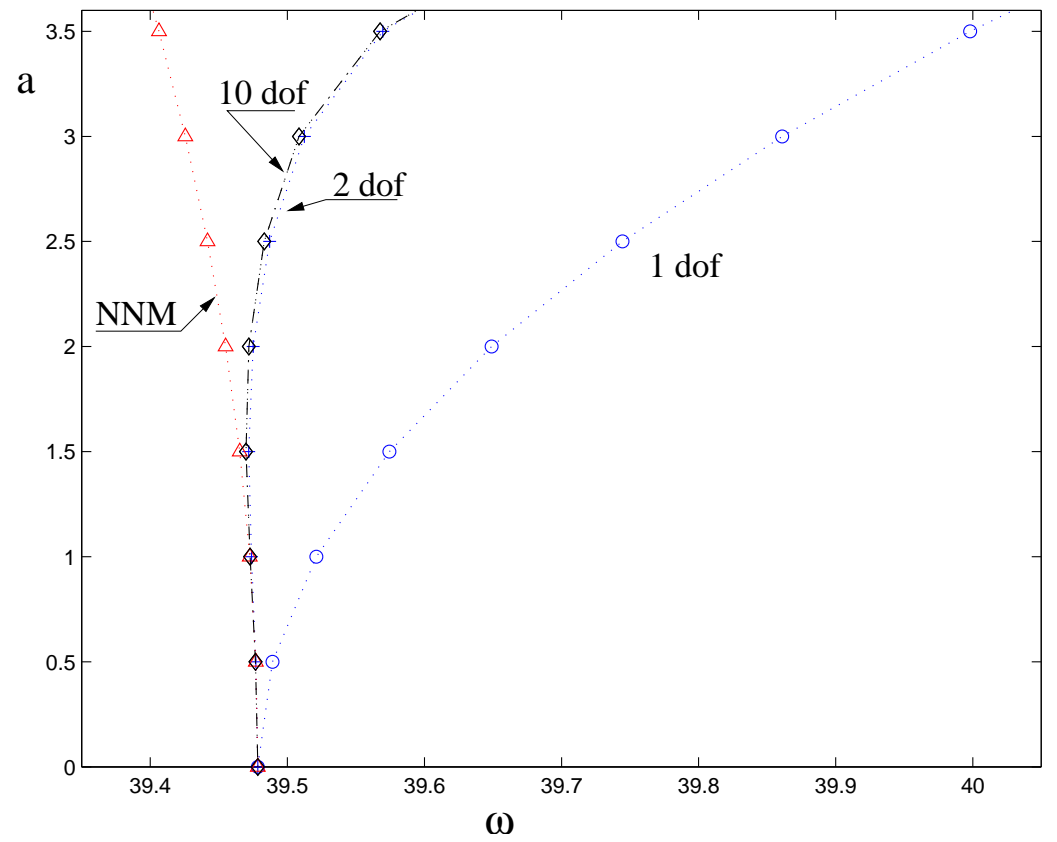

Figure 3. 


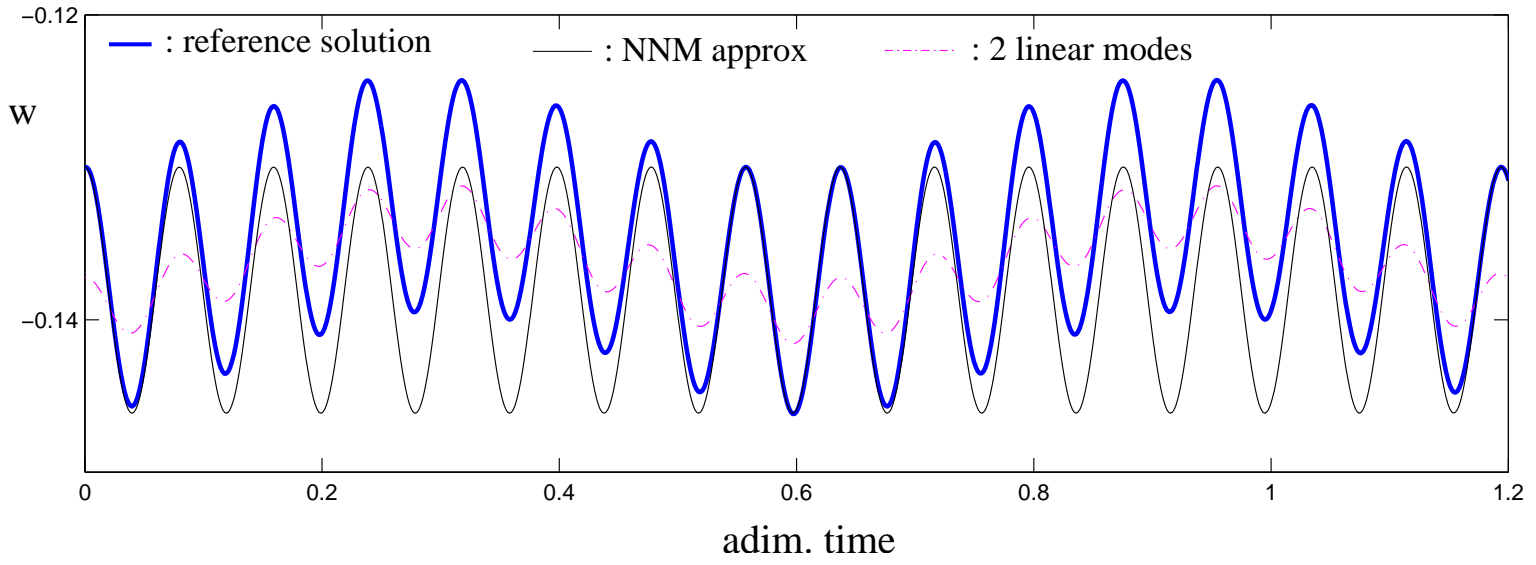

Figure 4. 

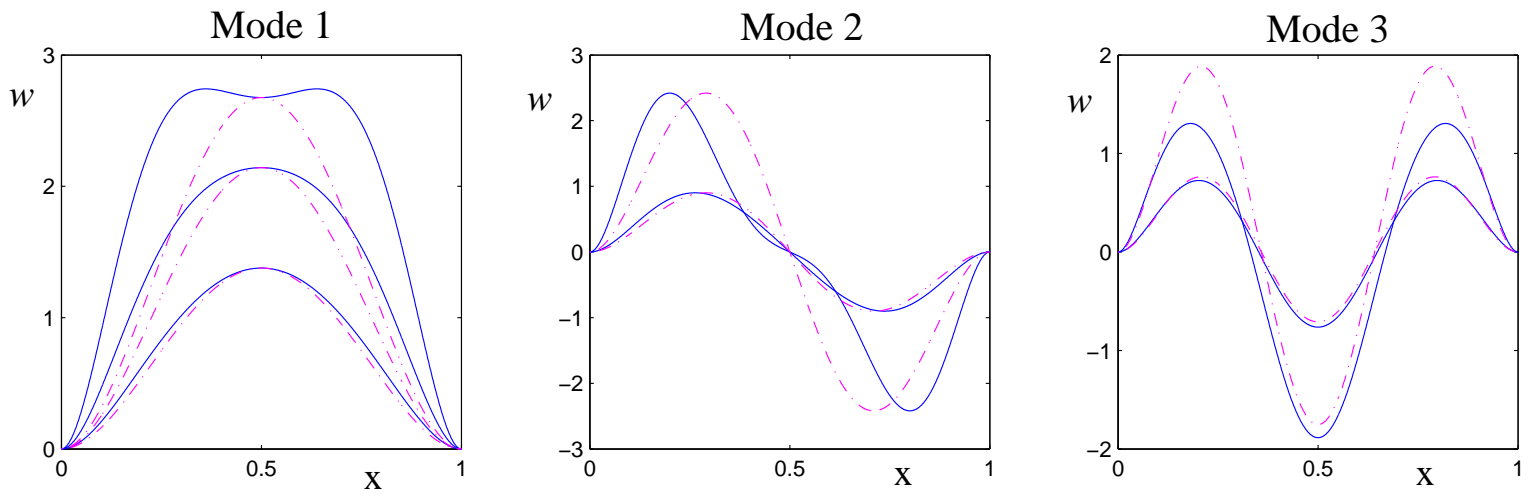

Figure 5. 

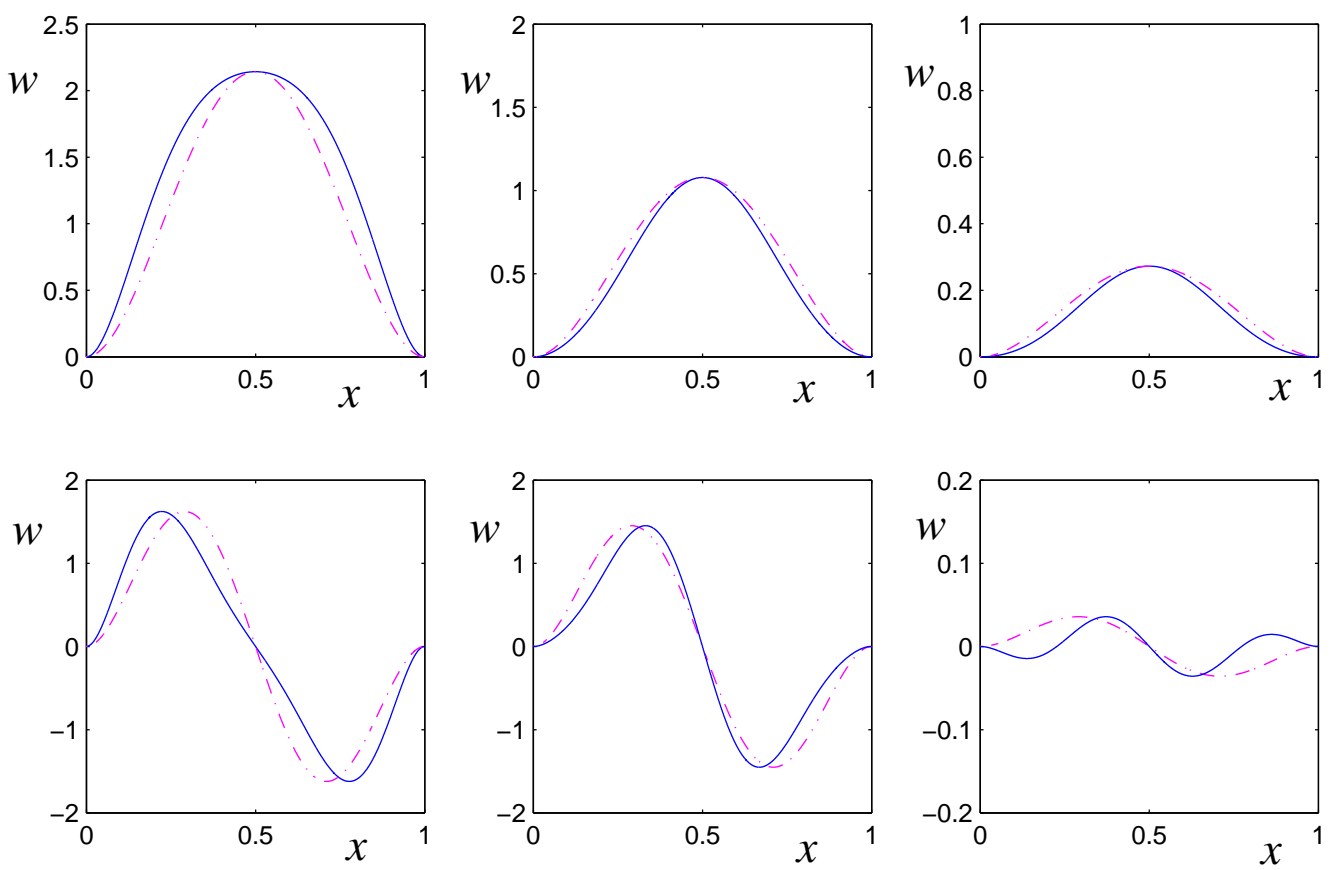

Figure 6. 


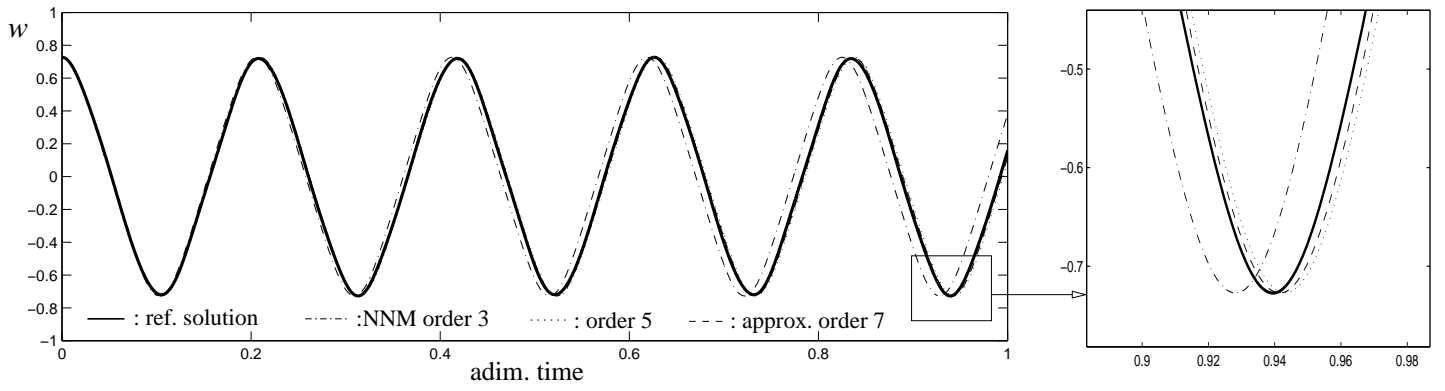

Figure 7. 

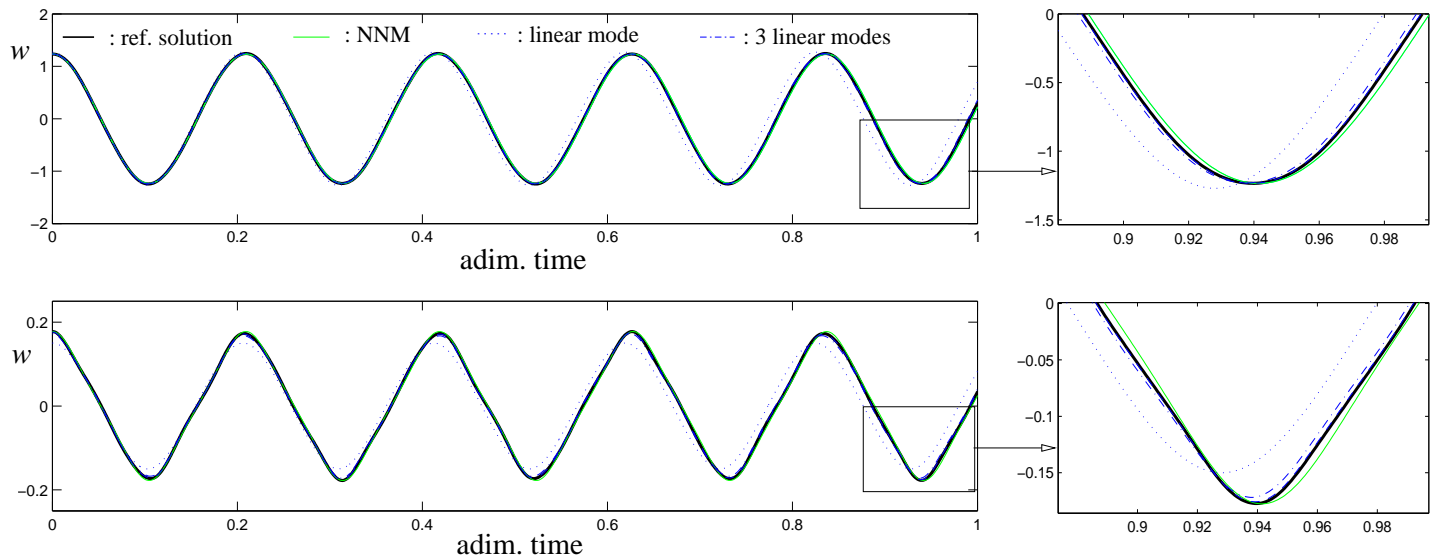

Figure 8 . 
36

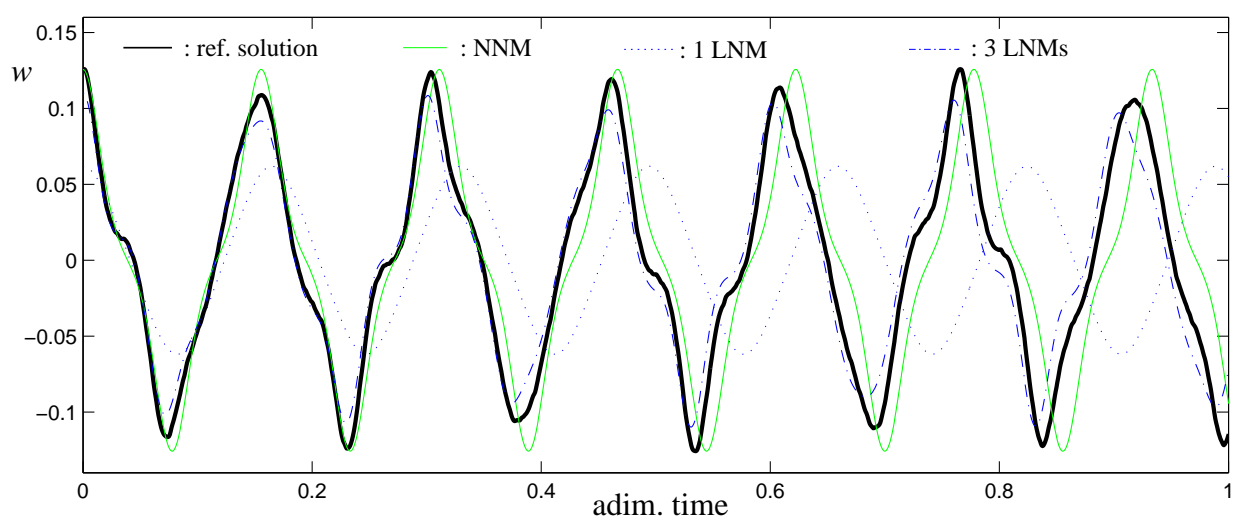

Figure 9. 

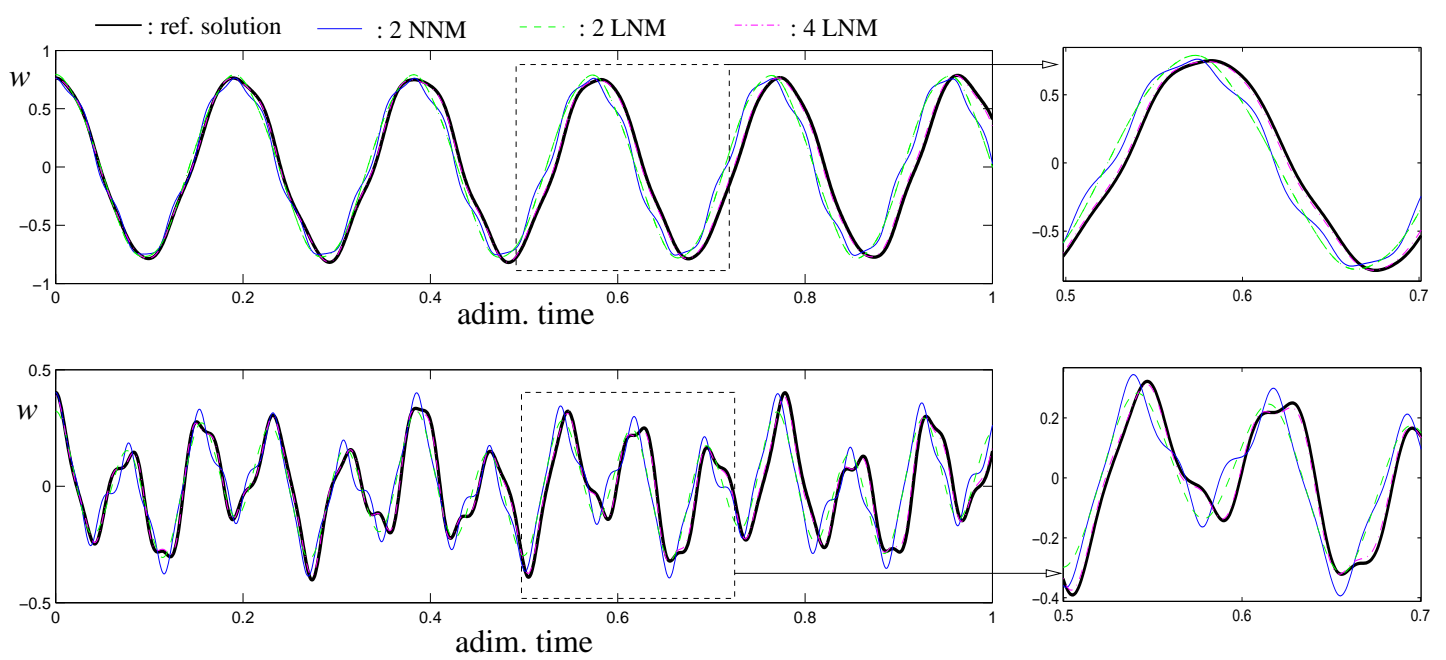

Figure 10. 
Dynamical system (physical co-ordinates)

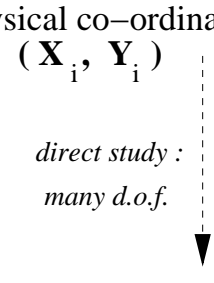

Non-linear change of

Normal dynamics

Study

Reduced-order

models

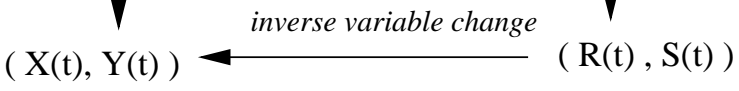

Figure 11. 